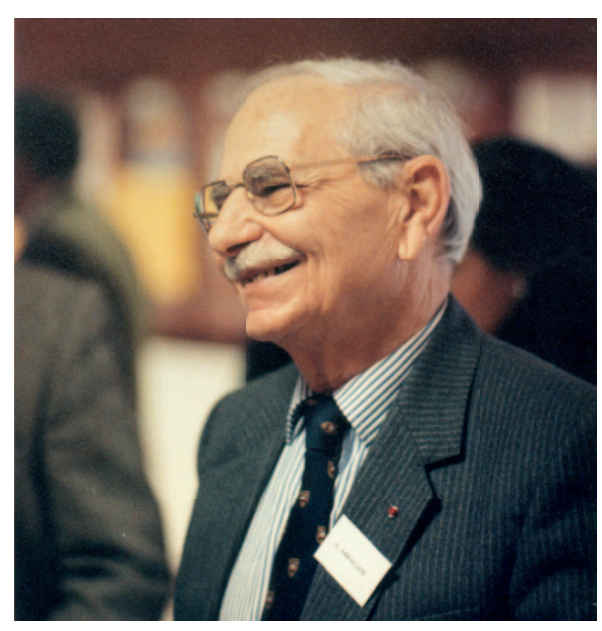

Anatole Abragam, au cours de la journée scientifique en l'honneur de ses 80 ans, le 3 juin 1994 à l'Orme des Merisiers, Centre de Saclay.

\section{Anatole Abragam}

et la Société Française de Physique

Président de la SFP en 1967, Anatole Abragam est à l'origine de ses statuts actuels. Jusqu'aux années 80 , il a régulièrement participé de façon très constructive, faisant souvent preuve d'un humour décapant, aux réunions de notre Conseil d'administration.

\section{Livres}

Anatole Abragam a écrit trois livres scientifiques :

- The Principles of Nuclear Magnetism, Clarendon Press (1961),

- Electron Paramagnetic Resonance of Transition Ions (avec B. Bleaney), Oxford University Press (1970),

- Nuclear Magnetism: Order and Disorder (avec M. Goldman), 0xford University Press (1982).

Un autre livre réunit un ensemble d'essais sur la science et les scientifiques :

- Réflexions d'un physicien, (en français), Hermann (1983).

Enfin, trois livres sont des versions différentes (et non pas de simples traductions) de ses mémoires, en français, en anglais et en russe, respectivement : - De la physique avant toute chose, Odile Jacob (1987), De la physique avant toute chose? (nouvelle édition), Odile Jacob (2001),

- Time Reversal, an autobiography, 0xford University Press (1989),

- Physicien, où as-tu été? (en russe), Nauka (1991).

\section{Anatole Abragam} (15 décembre 1914 - 8 juin 2011)

Anatole Abragam, physicien français, russe de naissance, membre de l'Académie des sciences, a laissé une profonde empreinte dans le domaine de la résonance magnétique, aussi bien électronique (RPE) que nucléaire (RMN), par ses travaux et contributions et son rôle éminent de pédagogue, surtout en RMN, où il a apporté clarté et rigueur théorique. Un grand nombre des plus éminents scientifiques de ce domaine se considèrent comme ses élèves. Il a été Président de la Société Française de Physique en 1967.

Cet article est une esquisse de sa vie et de sa carrière.

Anatole Abragam est né en Russie le 15 décembre 1914. Son père possédait une petite entreprise artisanale, et sa mère était docteur en médecine, après des études à Berne. Les difficultés croissantes de la vie en URSS les ont conduits à émigrer en France en 1925. Il y partit avec sa mère et sa sœur, mais son père fut empêché de partir et ne put les rejoindre que onze ans plus tard.

Après de brillantes études secondaires au lycée Janson-de-Sailly, dans l'embarras du choix d'une voie d'études supérieures, il opta pour la médecine, la profession de sa mère. Il perdit un an à réaliser qu'il n'y avait aucun goût, puis se tourna vers les mathématiques et la physique à la Faculté des Sciences, où il obtint une Licence-ès-Sciences en 1936. Il perdit les trois années suivantes à chercher un patron convenable pour préparer une thèse de Doctorat, jusqu'à ce que le déclenchement de la Seconde Guerre mondiale se charge de l'occuper pendant les années suivantes. Mobilisé, puis démobilisé après la débâcle, sans avoir jamais vu l'ennemi, il se réfugia en zone libre, à Saint-Raphaël, où il resta trois années, et subsista comme enseignant dans des établissements privés, professeur de latin, puis de physique, puis même de gymnastique. Il se réfugia ensuite à Grenoble, puis dans divers endroits discrets des Alpes, et s'engagea enfin dans l'armée par l'intermédiaire des FFI, où il passa une année.

En 1945, âgé de trente ans, sans profession et sans grands diplômes, il reprit des études à l'École Supérieure d'Électricité, d'où il sortit en 1947 Ingénieur en Radioélectricité. Il fut embauché la même année dans le tout jeune Commissariat à l'Énergie Atomique, initialement dans le Service de Physique Mathématique, composé de quatre membres, rapidement baptisés «les trois mousquetaires ». C'est le CEA qui l'envoya à l'Université d'Oxford, de 1948 à 1950, dans un laboratoire de RPE où, sous la direction de Maurice Pryce, il acquit un renom mondial dans le domaine, par sa contribution à la théorie de l'Hamiltonien de spin et sa théorie originale de la polarisation du cœur électronique, qui résolvait des problèmes paradoxaux d'interactions hyperfines, et pour laquelle il obtint un $\mathrm{PhD}$. Après un séjour à l'Université Harvard aux États-Unis en 1952-53, il devint un spécialiste de la RMN et fut à nouveau mondialement reconnu pour l'élaboration, avec Robert Pound, de la théorie des corrélations angulaires perturbées d'une cascade de deux rayonnements gamma. 


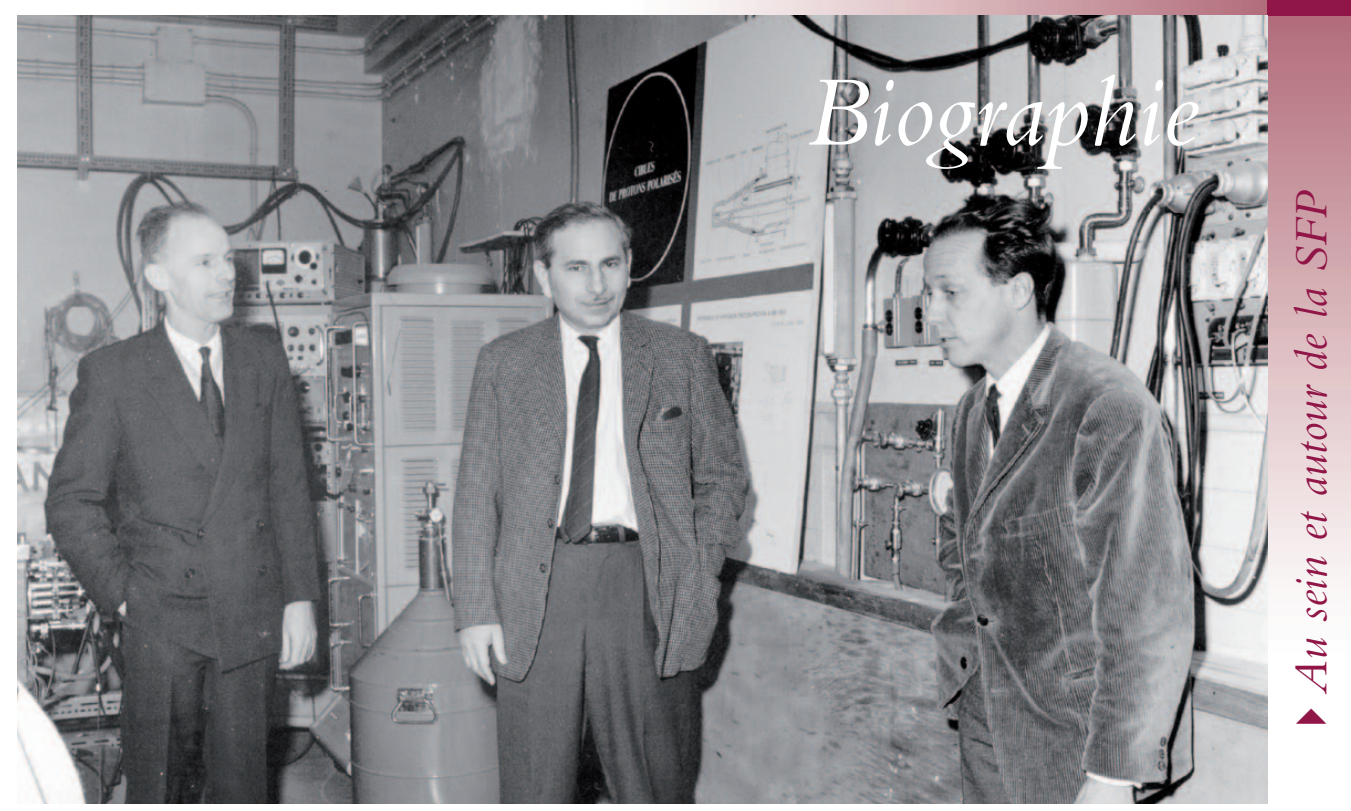

Anatole Abragam en 1962, avec Pierre Roubeau, cryogéniste (à gauche), et Charles Ryter, spécialiste de RPE (à droite), lors de la préparation de la première expérience de physique des particules avec cible de protons polarisés au CERN.

De retour au CEA, il créa en 1955 son Laboratoire de Résonance Magnétique, épaulé par Ionel Solomon pour la RMN et Jean Combrisson pour la RPE. Il devait le diriger pendant trente ans. Ionel Solomon, depuis membre de l'Académie des sciences, président de la SFP en 1973 et 1974, a eu un rôle fondamental dans cette création, pour en faire un véritable laboratoire expérimental, en même temps que théorique, alors qu'Abragam était un théoricien pur, quoique doué d'un sens précis de ce que devait être une expérience, dans tous ses détails. Les quelques années qui suivirent ont vu une véritable explosion de travaux prestigieux qui ont fait date, et grâce auxquels on a qualifié ce laboratoire de "plus productif du monde", en particulier :

- réalisation d'un magnétomètre à champ terrestre sous la forme d'un auto-oscillateur à la fréquence de Larmor des protons de l'eau, qui a rendu et rend encore de grands services pratiques ; - travaux conceptuels et expérimentaux sur la température de spin en champ magnétique faible dans les solides (et compréhension et popularisation de concepts analogues développés par un collègue peu auparavant, en champ élevé et sous irradiation de radiofréquence) ;

- invention et mise en évidence expérimentale de la polarisation dynamique nucléaire dans les solides isolants, en particulier pour produire des cibles polarisées pour la physique nucléaire et la physique des particules ;

- conception du principe de production d'ordre magnétique nucléaire, dont la polarisation dynamique est une étape, mis en évidence et étudié dans le laboratoire pendant deux décennies. Par la suite, sans compter les nombreux travaux d'autres membres du laboratoire, qu'il a suscités, conseillés ou suivis, Anatole Abragam a, à son actif, entre autres, deux contributions phares à la physique :

- le "pseudo-magnétisme " des neutrons, dû à leur interaction forte avec les spins nucléaires, et ses corollaires les moments " pseudo-magnétiques » nucléaires, la résonance et la précession "pseudo-magnétiques ", permettant leur utilisation pour l'étude des structures magnétiques nucléaires par diffraction des neutrons ;

- la spectroscopie des muons par croisement de fréquences, qui a profondément marqué ce domaine en modifiant totalement la pratique de cette spectroscopie.
C'est cependant à son rôle de pédagogue et d'enseignant hors pair qu'il doit son plus grand renom. D'abord auprès des membres du laboratoire, dont il a assuré la formation la plus rigoureuse et la plus enrichissante : il allait voir chacun chaque jour à sa table de travail et l'interrogeait dans les moindres détails sur son travail, jusqu'à le lui faire voir sous un jour nouveau ; il " épluchait » chaque manuscrit, le critiquait de façon constructive et en faisait corriger pratiquement chaque phrase. Son enseignement a commencé par des cours de RMN à Saclay, suivis par toute la jeune génération de physiciens et qui les a éblouis par leur profondeur, leur rigueur et leur clarté. Il est devenu Professeur au Collège de France en 1960, sous l'impulsion d'Alfred Kastler, car, dépourvu d'un Doctorat d'État issu d'une université française, il ne pouvait devenir professeur d'université. Oxford, connais pas! Il a enseigné au Collège jusqu'à sa retraite en 1985. Parmi ses livres, celui de 1961, The Principles of Nuclear Magnetism, a connu un tel succès mondial qu'il a un peu éclipsé ses autres travaux, ce qu'il a toujours regretté.

Anatole Abragam a assumé de nombreuses responsabilités hiérarchiques au sein du CEA : Chef de Section, Chef de Service, Chef de Département, puis Directeur de la Physique, de 1965 à 1970, poste dans lequel il s'est profondément impliqué, pour le plus grand bénéfice de la communauté des physiciens français, au détriment de ses propres travaux durant cette période, un prix accepté.

Il était membre de l'Académie des sciences depuis 1973, membre étranger de l'American Academy of Arts and Sciences (1974), de la National Academy of Sciences américaine (1977), de la Royal Society (1983), de l'Académie des Sciences de Russie (1999), Docteur honoris causa des Universités de Kent et d'Oxford au Royaume Uni et du Technion de Haïfa en Israël, Honorary Fellow de trois collèges d'Oxford : Merton, Jesus et Magdalen, couronné de nombreux prix scientifiques, médailles et distinctions prestigieuses, dont le prix Holweck en 1958. Il était Commandeur de la Légion d'honneur, Grand Croix de l'Ordre national du mérite, et Commandeur de l'Ordre des palmes académiques.

De notre point de vue, son plus grand titre reste qu'il a été un bon physicien.

Anatole Abragam est décédé le 8 juin 2011, dans sa 97e année. I

Maurice Goldman (maurice.goldman@free.fr) Membre de l'Académie des sciences 